\title{
Delay Distribution Analysis of IEEE 802.11 with Variable Packet Length
}

\author{
P. Raptis, V. Vitsas \\ Information Technology \\ TEI Thessaloniki, Greece \\ \{praptis, vitsas\}@it.teithe.gr
}

\author{
A. Banchs \\ Ingenieria Telematica \\ Univ. Carlos III de Madrid, Spain \\ banchs@it.uc3m.es
}

\author{
K. Paparrizos \\ Applied Informatics \\ Univ. of Macedonia, Greece \\ paparriz@uom.gr
}

\begin{abstract}
The distribution of the delay is a very important measure to determine the performance of a WLAN. Indeed, realtime applications typically require that their packets reach their destination within a certain delay with a given probability, and to guarantee this it is necessary to perform the computation of the delay distribution. In this paper we develop a novel method to compute the delay distribution of IEEE 802.11 Distributed Coordination Function (DCF) for a WLAN where the stations transmit packets of variable length. We develop an analytical model that applies to basic, RTS/CTS, and hybrid (coexistence of basic and RTS/CTS) access modes. A simulation model is used to validate the analysis.
\end{abstract}

Index Terms-IEEE 802.11; Performance; Delay distribution; Variable packet lengths.

\section{INTRODUCTION}

The most deployed protocol for Wireless Local Area Networks (WLANs) worldwide is the IEEE 802.11. IEEE 802.11 specifies a Medium Access Control (MAC) protocol in order to share the medium [1]. MAC employs the Distributed Coordination Function (DCF) for contention-based channel access which uses the Binary Exponential Backoff (BEB) algorithm to minimize collision probability. DCF provides two techniques to transmit data packets; the basic access and the Request-To-Send/Clear-To-Send (RTS/CTS) access method.

A lot of research has been carried out in studying analytically different performance metrics of the DCF. Bianchi [2] proposed a two-dimensional Markov chain model to calculate the saturation throughput performance considering infinite number of packet retransmissions. Wu [3] modified the model of [2] taking into account the packet's retransmission limit specified in the standard [1]. Chatzimisios [4] developed a model that calculates the average packet delay. In [5] we suggested a model that calculates the mean packet delay for a specific a number of retransmissions.

Utilizing z-transform of the packet delay, [6],[7] and [8] calculated the probability generating function (pgf) which in turn is used to compute the probability distribution function (pdf) of the packet delay. The method employed by [6],[7] and [8] is computationally very expensive. Author in [9] proposed an effective model that computes the probability of a

The project is co-funded by the European Social Fund \& Greek National Resources - EPEAEK II - ARCHIMIDIS. packet to be successful transmitted with delay time lower than a given value. Although less complex than [6],[7] and [8], the method of [9] still involves a number of relatively expensive operations. In [10] we proposed a simple, accurate and effective delay distribution analysis for basic access mode while in [11] for RTS/CTS mechanism. The model in [11] is also accurate, effective but more complex than [10].

The distribution of the delay is a very important measure to determine the performance of a WLAN. Indeed, real-time applications typically require to deliver packets in a timely fashion so as to meet application's requirements, it is necessary to have a good understanding of WLAN delay distribution. Previous analyses of the delay distribution [6-11] are commonly based on the unrealistic assumption of fixed length packets, which prevents their use to analyze realistic WLAN environments. In this paper we extend [11] and develop a novel method to compute the delay distribution of a WLAN under variable packet lengths. We develop an analytical model that applies to basic, RTS/CTS and hybrid access (coexistence of basic and RTS/CTS mechanisms).

The rest of the paper is organized as follows. Section II briefly describes DCF. Section III describes the mathematical model of the DCF. Section IV presents the delay distribution analysis. Section V describes the variable packet length analysis of the proposed model. Section VI presents the delay distribution with variable packet length analysis. Section VII validates the model by comparing analytical to simulative results and, finally, Section VIII presents the conclusions.

\section{Distributed COORDINATION FUnCTION}

\section{A. Basic access}

Each station senses the channel before transmitting a packet. If the channel is idle for a period of Distributed Inter Frame Space (DIFS) then the station transmits its packet. If the channel is busy, the station defers until an idle DIFS is detected and then generates a random backoff interval before transmitting. The random backoff interval is not arbitrary; rather it is a random, integer multiple of a particular slot time. This multiple is determined by the integer backoff counter. The backoff time counter is decreased as the channel is sensed idle, is stopped when the channel is busy and is resumed when the channel is sensed idle again for more than DIFS. A station transmits the packet when its backoff timer reaches zero. If the destination station successfully receives the packet, it waits for 
a short inter-frame space (SIFS) time interval and replies with an acknowledgement (ACK) packet. If the transmitting station does not receive an ACK frame from the receiver within a specified ACK timeout interval, the data packet is assumed lost and the station schedules a retransmission.

The random backoff value is selected in the range $\left[0, W_{i}\right]$, where $W_{i}$ is the current contention window $(\mathrm{CW})$ size and $i$ is the number of retransmissions (backoff stage number). The $C W$ is set to minimum value $C W_{\min }=W_{0}=W$ at the first packet transmission or after a successful transmission; after an unsuccessful transmission the $C W$ is doubled up (to a maximum value $C W_{\max }=W_{m}=2^{m} \mathrm{~W}$, where $m$ is the number of different $C W$ sizes) and a retransmission is scheduled. If the total number of transmission attempts for a packet reaches the retry limit $R$, the packet is dropped and the $C W$ is set to $C W_{\min }$.

\section{B. RTS/CTS access}

In order to cope the hidden terminal problem and to reduce the collision duration the RTS/CTS access scheme is used. The RTS/CTS access follows the same backoff rules as basic access. When the backoff timer reaches zero, the station sends a short RTS packet first instead of the data packet. The receiving station responds with a CTS packet after a SIFS time interval. The sender is allowed to transmit the data packet only if it receives a valid CTS. Upon the successful reception of the data packet the receiver transmits an ACK frame.

\section{Hybrid access}

In the hybrid access the packets are transmitted using basic or RTS/CTS access scheme. If a packet exceeds in size a given predetermined threshold value then the RTS/CTS access is used otherwise the packet is transmitted by means of basic access scheme.

\section{DCF MATHEMATICAL Model}

In this study we assume that the channel conditions are ideal (no transmission errors, no hidden stations and no capture effect), the number of contending stations $n$ is fixed and each station has always a packet available for transmission (i.e., the station works in saturated conditions).

Let $b(t)$ and $s(t)$ be the stochastic processes representing the backoff time counter and the backoff stage $(0, \ldots, R)$ respectively for a given station at time $t$. The two-dimensional process $\{s(t), b(t)\}$ is a discrete-time Markov chain. In this study we utilize the Markov chain model of [3]. The key approximation in that model is that each packet transmission collides with constant and independent probability $p$ regardless of the backoff stage. The probability $\tau$ that a station transmits a packet in a randomly chosen slot time can be expressed as [3]:

$$
\tau=\frac{2 \cdot(1-2 p) \cdot\left(1-p^{R+1}\right)}{W \cdot\left(1-(2 p)^{m+1}\right) \cdot(1-p)+(1-2 p) \cdot\left[\left(1-p^{R+1}\right)+W \cdot 2^{m} \cdot p^{m+1} \cdot\left(1-p^{R-m}\right)\right]}, R \geq m
$$

The probability $p$ that a transmitted packet encounters a collision is given by:

$$
p=1-(1-\tau)^{n-1}
$$

Equations (1) and (2) represent a non-linear system with two unknown values, $\tau$ and $p$, which can be solved using numerical methods and has a unique solution.

Let $q_{t r}$ be the probability that at least one station (out of $n$ ) transmits in a considered slot time:

$$
q_{t r}=1-(1-\tau)^{n}
$$

Let $q_{s}$ be the probability that a transmission occurring on the channel is successful. This can be computed as the probability that only one station transmits and the $n-1$ remaining stations do not, given the condition that a transmission occurs on the channel. Probability $q_{s}$ is given by:

$$
q_{s}=\frac{n \cdot \tau \cdot(1-\tau)^{n-1}}{q_{t r}}
$$

Let $E[s l o t]$ be the average time for the backoff counter to decrement by one. $E[$ slot $]$ is given by:

$$
E[s l o t]=\left(1-q_{t r}\right) \sigma+q_{t r} q_{s} T_{s}+q_{t r}\left(1-q_{s}\right) T_{c}
$$

where $\sigma$ is the duration of an empty slot, and $T_{s}$ and $T_{c}$ are the time durations the channel is sensed busy during a successful transmission and a collision, respectively.

Some parts of the Ts and Tc headers can be transmitted at different bit rates. The MAC headers,

The time duration of $T_{s}$ and $T_{c}$ depends on the channel access method employed. For the basic access method, we have:

$$
T_{s}^{b a s}=T_{c}^{b a s}=O^{b a s}+\frac{l}{C}
$$

where $l$ is the packet length and $O^{b a s}$ is the packet overhead

$$
O^{\text {bas }}=D I F S+\frac{H}{C}+2 \delta+S I F S+\frac{A C K}{C}
$$

$H$ is the packet header size (equal to the sum of MAC and physical headers), $\delta$ is the propagation delay and $C$ is channel bit rate.

For RTS/CTS method we have:

$$
\begin{gathered}
T_{s}^{r t s}=O^{r t s}+\frac{l}{C} \\
T_{c}^{r t s}=D I F S+R T S+S I F S+C T S
\end{gathered}
$$

where $O^{\text {ts }}$ is the packet overhead

$$
O^{r t s}=D I F S+R T S+3 S I F S+C T S+\frac{H}{C}+\frac{A C K}{C}
$$

\section{Delay Distribution}

The packet delay is defined as the time interval elapsed between the moment the packet is at the head of the MAC queue and the time when an ACK for this packet is received. The packet delay distribution is a discrete distribution whose the smallest time unit of the backoff counter is one time slot [7].

The first step of our mathematical model is to group packet time delays according to the number of collisions suffered by a packet. Note that this number corresponds to the stage at which the successful transmission occurs [5].

The delay $D$ of a packet successfully transmitted at backoff stage $j$ takes a value of the form: 


$$
D=T_{s} N_{s}+T_{c} N_{c}+N_{e} \sigma+j T_{c}+T_{s} \quad \text { for } 0 \leq j \leq R
$$

where $N_{e}$ is the number of empty slots that a packet encounters before its successful transmission, $N_{s}\left(N_{c}\right)$ is the number of successful (collided) transmissions from the rest of the stations that a packet encounters before its successful transmission, $j T_{c}$ is the time that the packet occupies the channel with collisions until it reaches the $j$ stage and $T_{s}$ is the time to transmit successfully from the $j$ th stage.

The key assumption upon which we base our analysis is the following one: we assume that the average relationship between $N_{s}, N_{c}$ and $N_{e}$ is a fixed one equal to the average, i.e.

$$
\frac{N_{e}}{P_{e}}=\frac{N_{s}}{P_{s}}=\frac{N_{c}}{P_{c}}
$$

Note that, on average, for every $P_{t r}$ slot times with a transmission (successful $P_{\mathrm{s}}$ or unsuccessful $P_{\mathrm{c}}$ ), we have $P_{e}$ empty slot times, where $P_{t r}$ is the probability with that at least one station out of $n-1$ transmits in a considered slot time, and $P_{e}$ is the probability that the channel is idle and are given by:

$$
\begin{gathered}
P_{t r}=1-(1-\tau)^{n-1}, \text { and } P_{e}=1-P_{t r} \\
P_{s}=(n-1) \cdot \tau \cdot(1-\tau)^{n-2} \text {, and } P_{c}=P_{t r}-P_{s}
\end{gathered}
$$

Using (11), (12), and (13) we rewrite $D$ as:

$$
D=N_{s}\left(T_{s}+T_{c}\left(P_{c} / P_{s}\right)+\sigma\left(P_{e} / P_{s}\right)\right)+j T_{c}+T_{s}
$$

From the above, we can express $D$ as a function of one variable: $N_{\mathrm{s}}$.

$$
N_{s, j}=\text { round_int }\left(\frac{D-j T_{c}-T_{s}}{T_{s}+\left(P_{c} / P_{s}\right) T_{c}+\left(P_{e} / P_{s}\right) \sigma}\right)
$$

From (15) we can compute the approximated $N s$ for a given delay $D$ :

$$
N_{s}=\text { round_int }\left(\frac{D-T_{s}}{T_{s}+\left(P_{c} / P_{s}\right) T_{c}+\left(P_{e} / P_{s}\right) \sigma}\right)
$$

The probability $P$ that a successfully transmitted packet encounters $N_{\mathrm{s}}$ transmissions is:

$$
P\left(N_{s}\right)=\sum_{j=0}^{R} P_{j}\left(N_{s}\right)
$$

where $P_{j}$ is the probability that the successful transmission occurs at stage $j$. A detailed analysis that computes $P_{j}$ can be found in [11] and is not presented here due to space limitations.

\section{VARiable Packet Length}

We consider that a packet length takes a value $l$ of the set $L$ with probability $P_{L}(l)$, where $L$ is the set of all possible packet lengths with corresponding probabilities in the set $P_{L}$. For simplicity, we assume that all stations pick the packet length from the same distribution $\left(l, P_{L}(l)\right)$ (the analysis would be very similar in the case when the stations pick the packet length from different distribution sets).

Let $E[l]$ be the average packet length of all possible $l$ in the set $L . E[l]$ is computed as following:

$$
E[l]=\sum_{l \in L} l \cdot P_{L}(l)
$$

Let $P_{\mathrm{k}}$ be the probability that exactly $k$ stations are involved in one collision:

$$
P_{k}=\left(\begin{array}{l}
n \\
k
\end{array}\right) \cdot \frac{\tau^{k}(1-\tau)^{n-k}}{q_{t r}\left(1-q_{s}\right)} \quad \text { for } k \geq 2
$$

Let $P_{\mathrm{y}}$ be the sum of probabilities that corresponds to packet lengths that are shorter or equal to the longest data packet $l$ in a collision:

$$
P_{y}=\sum_{h \in L \mid h<l} P_{L}(h)
$$

Let $P_{l, k}$ be the probability that a packet with length $l$ is the longest data packet in a collision when $k$ stations are involved in a collision:

$$
P_{l, k}=\sum_{r=1}^{k}(-1)^{r+1}\left(\begin{array}{l}
k \\
r
\end{array}\right)\left(P_{L}(l)\right)^{r} P_{y}^{k-r} \quad \text { for } 2 \leq k \leq n
$$

From (21) we can compute the probability that any number of stations can be involved in one collision.

Let us now compute the time duration of $T_{s}$ and $T_{c}$ for the different access schemes.

\section{A. Basic Access}

The time $T_{s}$ for a successful transmission is the sum of packet's overhead $O^{\text {bas }}$ plus the average payload:

$$
T_{s}=O^{\text {bas }}+\frac{1}{C} \cdot E[l]
$$

In the basic access collisions occur between data packets. The time duration of a collision is the time of the longest data packet involved in a collision:

$$
T_{c}=O^{\text {bas }}+\frac{1}{C} \cdot \sum_{l \in L}\left(l \cdot \sum_{k=2}^{n} P_{k} P_{l, k}\right)
$$

\section{B. RTS/CTS Access Mode}

In the RTS/CTS scheme the computation of $T_{s}$ is similar to that of basic access, while a collision last $T_{c}^{r t s}(9)$ :

$$
\begin{gathered}
T_{s}=O^{r t s}+\frac{1}{C} \cdot E[l] \\
T_{c}=T_{c}^{r t s}
\end{gathered}
$$

\section{Hybrid Access}

The times $T_{s}$ and $T_{c}$ in hybrid access depend on the RTSthreshold value $l_{T H}$. If the data packet is less than the threshold then basic access is used otherwise RTS/CTS scheme is used. The $T_{s}$ time is computed as following:

$$
T_{s}=O^{b a s} \sum_{l \leq l_{T H}} P_{L}(l)+O_{s}^{r t s} \sum_{l>>_{T H}} P_{L}(l)+\frac{1}{C} \cdot E[l]
$$

In the hybrid access mode may occur three possible collision scenarios: 1) collision between data packets, 2) collision between RTS frames and data packets, and 3) collision between RTS frames. In the first and the second scenario the collision depends on the longest data packet involved in the collision as the packet header $H$ is always higher than the length of a RTS frame [1]. In the third scenario the duration of a collision is the time of a RTS frame. The time collision is computed as: 


$$
T_{c}=O^{\text {bas }} \sum_{l \leq l_{T H}} P_{L}(l)+\frac{1}{C} \cdot \sum_{l \leq l_{T H}}\left(l \cdot \sum_{k=2}^{n} P_{k} P_{l, k}\right)+T_{c}^{r t s} \sum_{l>l_{T H}}\left(\sum_{k=2}^{n} P_{k} P_{l, k}\right)
$$

\section{Delay Distribution With VARiable PaCKet LENGTH}

Let $P_{p}$ be the partial probability that a packet of length $l$ (of the set $L$ ) is successfully transmitted after experiencing delay $D$ is given by:

$$
P_{p}(l)=P_{L}(l) \cdot P\left(N_{s}\right)
$$

In order to compute $P_{p}$ for packet length $l$, we follow the next steps, 1) we decide the access mode for a WLAN, 2) we compute $T_{s}$ and $T_{c}$ for the chosen access mode (as described in Section V), 3) for a given delay $D$ we compute $N_{s}$ from (16), 4) we compute total probability $P$ from (17), and finally 5) substituting $P$ to (28) we compute the partial probability.

\section{VALIDATION AND RESUltS}

In order to validate our model we compare simulative to analytical results. The parameter values used for both simulation and analytical results follow the values specified for the Direct Spread Sequence Spectrum (DSSS) employed in the IEEE $802.11 \mathrm{~b}$ standard and are shown in Table I. The packet length $l$ is taken from the set $L=[1,5,10,12]$ Kbits with corresponding probabilities $P_{L}=[0.5,0.05,0.15,0.3]$.

Table I. System Parameter Values

\begin{tabular}{ll}
\hline \hline Channel bit rate & 1 Mbit/s \\
Packet Payload & Variable length \\
MAC header & 224 bits \\
PHY header & 192 bits \\
ACK & 112 bits + PHY header \\
RTS & 160 bits + PHY header \\
CTS & 112 bits + PHY header \\
Propagation delay, $\delta$ & $1 \mu \mathrm{s}$ \\
Slot time, $\sigma$ & $20 \mu \mathrm{s}$ \\
SIFS & $10 \mu \mathrm{s}$ \\
DIFS & $50 \mu \mathrm{s}$ \\
Minimum $W, W_{0}$ & 32 \\
Number of $W$ sizes, $m$ & 5 \\
Short retry limit, $R$ & 6
\end{tabular}

Fig. 1a, 1b, 1c plot the delay distributions for packet lengths of the set $L$ (partial probabilities vs. delay) for $n=25$ using hybrid, basic and RTS/CTS access respectively. The analytical results for figures $1 \mathrm{a}, 1 \mathrm{~b}, 1 \mathrm{c}$ are derived from (28). For the hybrid access we used threshold value $l_{T H}=1860$ bits. The model is accurate as the analytical fairly match the simulation results for all access modes. All simulation results are taken with a $95 \%$ confidence interval lower than 0.0001 . For basic access and given delay $1 \mathrm{sec}$ the (17) gives (total) probability 0.000602 while (28) gives the partial probabilities (plotted in Fig. 1b) 0.000301 ( $l=1$ Kbits), 0.000030 ( $l=5 \mathrm{Kbits}$ ), 0.000090 ( $l=10 \mathrm{Kbits}$ ), 0.000181 ( $l=12 \mathrm{Kbits})$. The results of the hybrid access in figure 1a and the RTS/CTS access in figure 1c are very similar as the threshold value we used in the hybrid access had little effect on the delay distributions for the chosen packet lengths (set $L$ ) and $P_{L}$ 's.

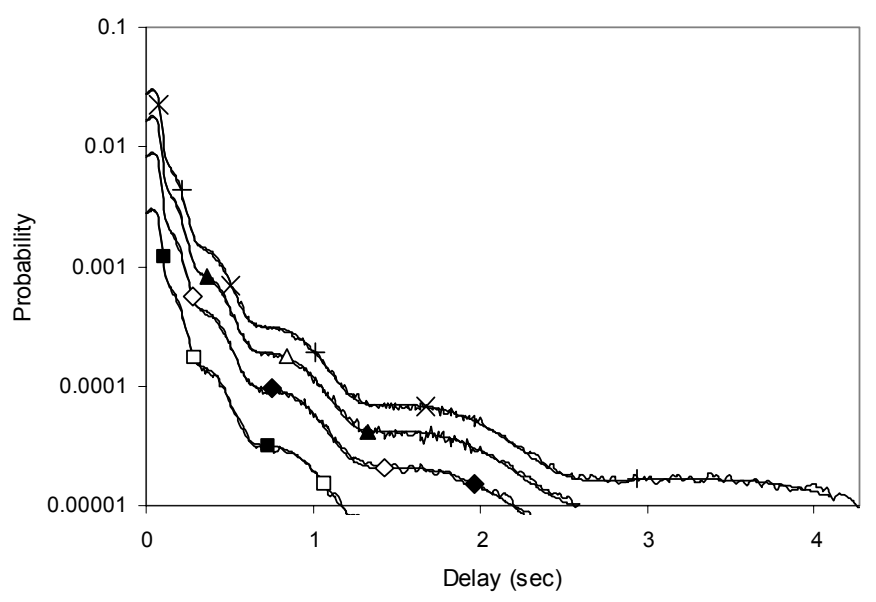

(a)

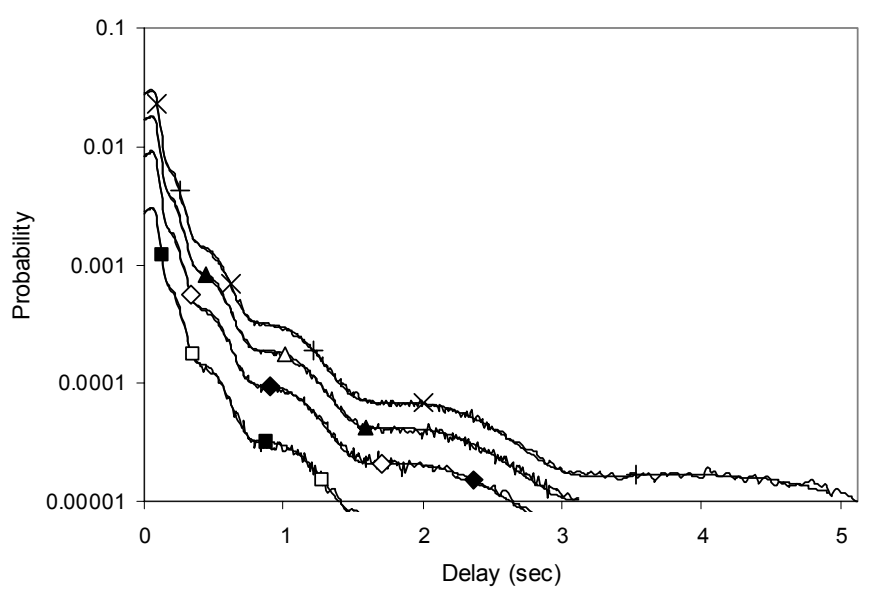

(b)

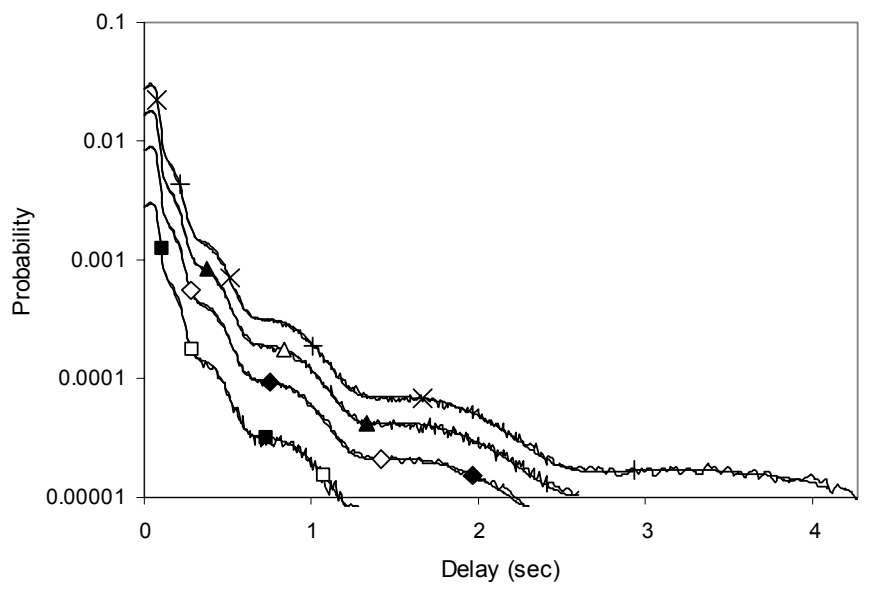

(c)

simulation, $l=5$ Kbits

$\square$ analytical, $l=5$ Kbits

- simulation, $l=10$ Kbits

$\Delta$ simulation, $l=12$ Kbits

+ simulation, $l=1$ Kbits

$\diamond$ analytical, $l=10 \mathrm{kbits}$

$\triangle$ analytical, $l=12$ Kbits

$\mathrm{x}$ analytical, $l=1 \mathrm{Kbits}$

Figure 1. Partial probabilities versus delay, (a) for hybrid access, (b) for basic access, and (c) for RTS/CTS access

If we zoom Fig. 1a in horizontal axis from 0.0-0.5 secs we get Fig. 2 (note that vertical axis is in linear scale). The figure 
confirms that the model is accurate as the simulative lines closely follow analytical lines.

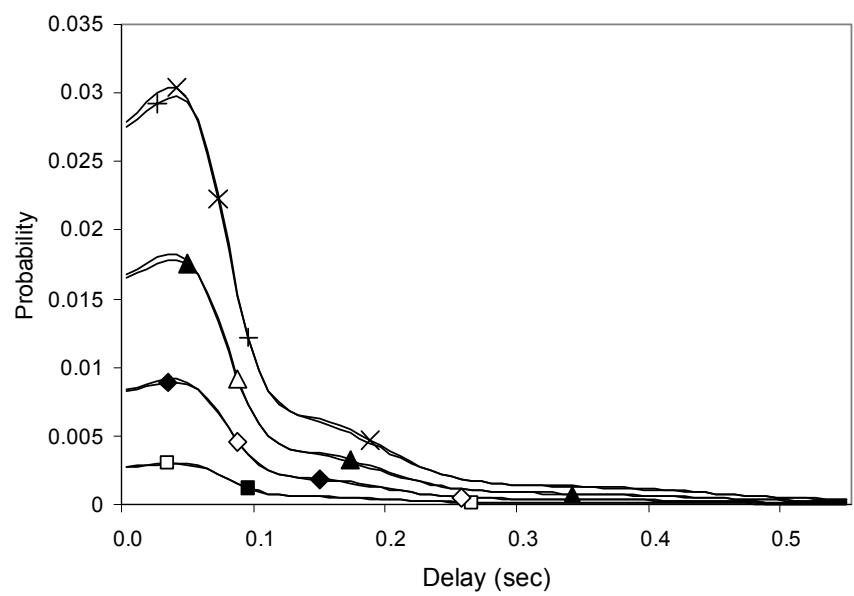

a simulation, $l=5 \mathrm{Kbits}$

- simulation, $l=10 \mathrm{Kbits}$

\ simulation, $l=12$ Kbits

+ simulation, $l=1$ Kbits

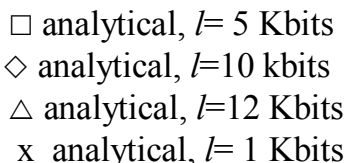

Figure 2. Partial probabilities versus delay for hybrid access

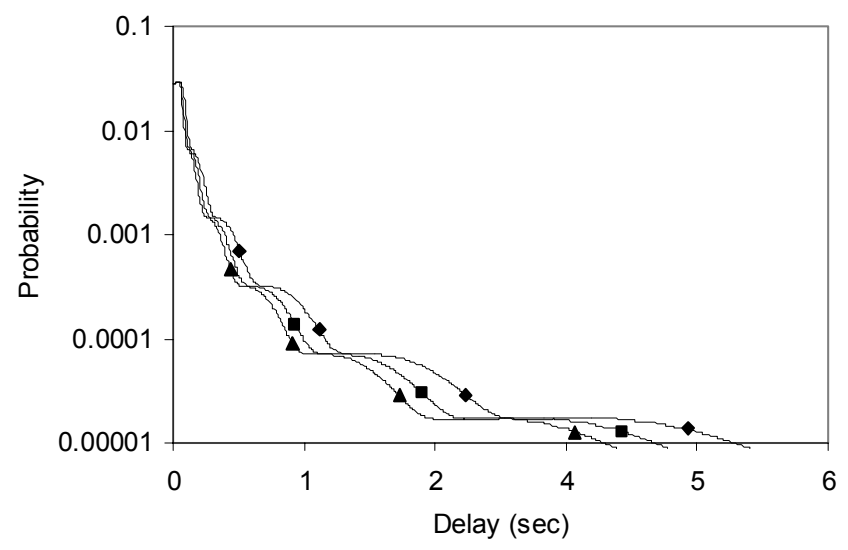

$\Delta$ RTS/CTS access, basic access, hybrid access

Figure 3. Delay distributions of $l=1 \mathrm{Kbits}$ for basic, RTS/CTS and hybrid access modes.

Fig. 3 plots analytical results of partial probabilities versus delay for packet lengths (only) of $l=1$ Kbits for three networks of $n=25$ each running in one of the three access modes basic, RTS/CTS and hybrid. From the figure we see that the probability to have a successful transmission of a packet of length $l=1 \mathrm{Kbits}$ is the same for all access modes but with different delay. For instance, if the probability to have a successful transmission is 0.0001 then the delays for basic, RTS/CTS, hybrid access would be $1.37 \mathrm{sec}, 1.05 \mathrm{sec}, 1.18 \mathrm{sec}$ respectively. In order to appear clearly the results for hybrid access we used a high threshold value $l_{T H}=10500$ bits.

\section{CONCLUSIONS}

The assumption that the stations in a WLAN transmit packets of variable sizes (such as voice and data packets) is more realistic than the assumption of fixed packet sizes. In this paper we develop a novel method to compute the delay distribution of IEEE 802.11 DCF for a WLAN where the stations transmit packets of variable length. We develop an analytical model that applies to basic, RTS/CTS, and hybrid access modes. Our model can compute the probability that a packet (of any length) will be successfully transmitted for a given delay value (the packet lengths are taken from a given distribution). The model is accurate as there is a good match between the simulation and analytical results.

\section{REFERENCES}

[1] IEEE standard for Wireless LAN Medium Access Control (MAC) and Physical Layer (PHY) specifications, IEEE Std: 802.11b-1999/Cor 1-2001, 2001.

[2] G. Bianchi, "Performance Analysis of the IEEE 802.11 Distributed Coordination Function", IEEE Journal on Selected Area in Communications, vol.18, no.3, pp. 535-547, 2000.

[3] H. Wu, Y. Peng, K. Long, S. Cheng, J. Ma, "Performance of Reliable Transport Protocol over IEEE 802.11 Wireless LAN: Analysis and Enhancement", Proc. of IEEE INFOCOM '02, pp.599-607, June 2002.

[4] P. Chatzimisios, A. C. Boucouvalas and V.Vitsas, "IEEE 802.11 Packet Delay - A Finite Retry Limit Analysis", IEEE GLOBECOM, vol. 2, pp.950-954, December 2003.

[5] P. Raptis, V. Vitsas, K. Paparrizos, P. Chatzimisios, and A. C. Boucouvalas, "Packet Delay Distribution of the IEEE 802.11 Distributed Coordination Function". IEEE Symposium on a World of Wireless, Mobile and Multimedia Networks (WoWMoM), pp. 299-304, 2005.

[6] O. Tickioo and B. Sikdar "Queueing Analysis and Delay Mitigation in IEEE 802.11 Random Access MAC based Wireless Networks", Proc. of IEEE INFOCOM '04, March 2004.

[7] H. Zhai and Y. Fang, "Performance of Wireless LANs Based on IEEE 802.11 MAC Protocols," IEEE Symposium on Personal, Indoor and Mobile Radio Communication (PIMRC), pp. 25862590, 2003.

[8] Hai L. Vu and Taka Sakurai, "Accurate Delay Distribution for IEEE 802.11 DCF” IEEE Communications Letters, vol. 10, no. 4, pp. 317-319, April 2006.

[9] A. Banchs, "Analysis of the Distribution of the Backoff Delay in 802.11: A Step Towards End-to-End Delay Guarantees in WLANs" QofIS 2004, LNCS 3266, pp.54-63, 2004.

[10] P. Raptis, A. Banchs, K. Paparrizos, "A Simple and Effective Delay Distribution Analysis for IEEE 802.11", 17th Annual IEEE International Symposium on Personal, Indoor and Mobile Radio Communications (PIMRC '06), 11-14 September 2006, Helsinki.

[11] P. Raptis, A. Banchs, V.Vitsas, P. Chatzimisios, K. Paparrizos, "Delay Distribution Analysis of the RTS/CTS mechanism of IEEE 802.11", 31st IEEE Conference on Local Computer Networks (LCN '06), 16-18 November 2006, Tampa, Florida, USA, pp. 404-410. 